\title{
Polysaccharide-mediated Protection Against Abscess Formation in Experimental Intra-Abdominal Sepsis
}

Arthur O. Tzianabos, ${ }^{*}$ Dennis L. Kasper, ${ }^{\star \ddagger}$ Ronald L. Cisneros, ${ }^{*}$ Roger S. Smith, ${ }^{\star}$ and Andrew B. Onderdonk ${ }^{\star \$}$

Channing Laboratory, Departments of *Medicine and ${ }^{\S}$ Pathology, Brigham and Women’s Hospital and ${ }^{\ddagger}$ Division of Infectious Diseases, Beth Israel Hospital, Harvard Medical School, Boston, Massachusetts 02115

\begin{abstract}
Abscess formation is a major complication of intra-abdominal sepsis that causes significant morbidity and mortality. In such cases, Bacteroides fragilis is the predominant anaerobic isolate. In a rat model of intra-abdominal sepsis, the capsular polysaccharide complex (CPC) from $B$. fragilis promotes abscess formation and when administered sub-cutaneously, protects against this host response by a $\mathbf{T}$ cell-dependent immune mechanism. In the present study, the polysaccharide A (PS A) component of CPC protected animals against challenge with live heterologous bacterial species (mixtures of anaerobes and facultative organisms) that are most commonly isolated from intra-abdominal abscesses in humans. Protection against heterologous bacterial challenge was transferred by $T$ cells. Administration of PS A shortly before or even after challenge with $B$. fragilis protected against this host response. In experiments designed to simulate fecal contamination of the human peritoneal cavity, PS A protected animals against abscess formation induced by a rat cecal contents inoculum. The surprisingly broad protective activity of PS A indicates that this molecule is likely suppressing a nonspecific host tissue reaction that forms in response to a variety of abscess-inducing organisms and that it might be useful in preventing abscess formation associated with intra-abdominal sepsis in the clinical setting. (J. Clin. Invest. 1995. 96:2727-2731.) Key words: intra-abdominal sepsis • Bacteroides fragilis • polysaccharide • abscess • immunomodulation
\end{abstract}

\section{Introduction}

Abscess formation is a distinct host response to a variety of invading microorganisms. In cases of intra-abdominal sepsis, this pathologic response may be initially beneficial to the host as a result of localization and containment of the bacterial insult within the peritoneal cavity. However, failure to treat fully formed abscesses causes severe morbidity in patients and can be fatal (1). Despite the use of antimicrobial therapy, surgical intervention is usually required to drain or remove established intra-abdominal abscesses.

Address correspondence to Dr. Arthur O. Tzianabos, Channing Laboratory, 180 Longwood Ave., Boston, MA 02115. Phone: 617-432-1610; FAX: 617-731-1541; e-mail: arttz@warren.med.harvard.edu

Received for publication 8 June 1995 and accepted in revised form 31 July 1995.

J. Clin. Invest.

(C) The American Society for Clinical Investigation, Inc. 0021-9738/95/12/2727/05 \$2.00

Volume 96, December 1995, 2727-2731
B. fragilis is the most frequently isolated anaerobic species from clinical cases of intra-abdominal sepsis $(2,3)$. Our studies have demonstrated that $B$. fragilis induces the formation of intra-abdominal abscesses associated with sepsis in animal models of disease $(4,5)$. Other species of Bacteroides, as well as anaerobes such as Fusobacterium varium that are associated with abscess formation in humans, required the presence of a facultative "helper" organism such as Enterococcus faecalis to induce abscesses in this system $(4,6,7)$. Studies performed to determine virulence factors associated with $B$. fragilis showed that the capsular polysaccharide complex $(\mathrm{CPC})^{1}$ from this organism induced abscesses that were histologically identical to those formed in response to intact organisms (5). In all studies, an adjuvant including sterile cecal contents and barium sulphate was required for abscess formation. While many anaerobes involved in intra-abdominal sepsis possess surface polysaccharides, the mechanisms by which these organisms induce abscesses during co-infection with facultative bacteria have not been determined.

The CPC of $B$. fragilis NCTC 9343 is composed of two surface expressed, high-molecular-weight component polysaccharides, termed PS A and PS B (8). Each polysaccharide is composed of oligosaccharide repeating units possessing constituent sugars with positively charged free amino groups as well as negatively charged carboxyl or phosphonate groups (9). The component polysaccharides in the CPC are aggregated by electrostatic interactions between oppositely charged groups (10). The elaboration of a complex capsular motif appears to be common among $B$. fragilis strains (11).

The positively and negatively charged groups on PS A and PS B mediate their ability to induce intra-abdominal abscesses (12). The presence of similar charged groups on polysaccharide repeating units from other bacteria (either in natural form or following chemical modification) also confers the ability to promote abscess formation (12). In addition to the capacity of $B$. fragilis CPC to facilitate abscess formation, prior subcutaneous administration of CPC in the absence of adjuvants affords protection against abscesses following intraperitoneal challenge with viable $B$. fragilis (13). Investigations of the structural determinants in the carbohydrate responsible for inducing protection against abscesses have shown that, as with the induction of abscesses, positively and negatively charged groups on otherwise distinct polysaccharides are critical to the ability to confer protection against abscess formation in the rat model (14).

Although bacterial polysaccharides are usually considered as $\mathrm{T}$ cell-independent antigens, a T cell-dependent mechanism mediates both abscess induction as well as protection against abscess formation in these animals $(6,14-16)$. The transfer of

1. Abbreviations used in this paper: $\mathrm{CPC}$, capsular polysaccharide complex; PS A, polysaccharide A. 
serum antibodies specific for the CPC failed to protect animals against abscess formation in these studies. The finding that $\mathrm{T}$ cells mediate abscess induction as well as protection has led us to consider that "protection" against heterologous abscessinducing polysaccharides may be secondary to an interruption in the circuit of cell-mediated immunologic events responsible for abscess induction. To address this question and to evaluate the potential use of this compound in humans, we tested whether the administration of PS A could confer protection against heterologous viable bacterial species commonly associated with abscess formation.

\section{Methods}

Bacterial strains and isolation of $B$. fragilis polysaccharide. $B$. fragilis NCTC 9343, B. distasonis 8503, and Fusobacterium varium ATCC 8501 were originally obtained from the National Collection of Type Cultures (London, England) or the American Type Culture Collection (Bethesda, MD). B. thetaiotaomicron 5482 and Enterococcus faecalis 2603 strains were obtained from the stock culture collection of the Channing Laboratory. Microorganisms were stored at $-80^{\circ} \mathrm{C}$ in peptone-yeast or brain heart infusion broth until used and grown anaerobically as previously described (8). The CPC from $B$. fragilis NCTC 9343 was isolated by hot phenol/water extraction and subsequent purification of PS A performed by isoelectric focusing $(8,10)$.

Animal model of intra-abdominal abscess formation. Briefly, male Wistar rats ( 180 to 200 grams, Charles River Laboratories, Wilmington, MA) were anesthetized with a single intraperitoneal injection of 0.15 $\mathrm{ml}$ of Nembutal $(50 \mathrm{mg} / \mathrm{ml}$; Abbott Laboratories, North Chicago, IL) (5). An anterior midline incision $(0.5 \mathrm{~cm})$ was made through the abdominal wall and peritoneum, and a gelatin capsule with $0.5 \mathrm{ml}$ of inoculum was inserted into the pelvis. The incisions were closed with silk sutures and animals returned to their cages. $6 \mathrm{~d}$ later, animals were necropsied in a blinded fashion and examined for intra-abdominal abscesses. The presence of one or more abscesses in an animal as defined previously (5) was scored as a positive result. Results are reported as a compilation of at least two separate experiments.

Preparation of challenge inocula. Inocula contained a 1:1 mixture of the challenge organism(s) and an adjuvant solution containing sterile rat cecal contents and $10 \%$ barium sulfate (wt/vol) as described (4). Bacteria were grown anaerobically and adjusted to the following concentrations (as determined by colony forming units on solid agar) per gelatin capsule: Bacteroides fragilis 9343-5 $\times 10^{7}, B$. distasonis-5 $\times 10^{7}, F$. varium- $2.5 \times 10^{7}$, B. thetaiotaomicron $5 \times 10^{7}$, E. faecalis $1.3 \times 10^{7}$. For some experiments, a cecal contents inoculum containing feces procured from the ceca of meat-fed rats was used to challenge animals (17). Quantitative and qualitative bacteriology of this inoculum was performed (17) and the results are shown in Table I. The cecal contents inoculum was mixed with barium sulfate ( $10 \%$ final concentration, wt $/ \mathrm{vol})$ and titered in the rat model to yield $\sim 50 \%$ mortality in a given group of rats with $100 \%$ rate of abscess formation in survivors.

Regimens for PS A administration. Animals were injected subcutaneously with $B$. fragilis PS A in $0.1 \mathrm{ml}$ of PBS. All treatment regimens are shown in Table II. For some experiments, animals were treated with PS A according to an extended regimen (regimen 1) comparable with that which had been previously established in protection studies using the CPC of $B$. fragilis (13). PS A was administered three times a week for $3 \mathrm{wk}$ with a booster dose $24 \mathrm{~h}$ before challenge. To determine the effect of shortening this treatment regimen, further experiments were performed in which regimen 1 was reduced a week at a time (regimens 1-3). A fourth regimen (regimen 4) included treatment with PS A 24 $\mathrm{h}$ before challenge as well as 4 and $24 \mathrm{~h}$ after challenge. For studying the protective effect of PS A against abscess formation when given after peritoneal contamination, a fifth protocol (regimen 5 ) was used in which animals were challenged with $B$. fragilis and treated with PS A 1, 24, and $48 \mathrm{~h}$ after surgery. Finally, to assess the efficacy of PS A against
Table I. Microbial Analysis of Cecal Contents Inoculum

\begin{tabular}{lcc}
\hline \multicolumn{1}{c}{ Organism } & CFU/ml ( $\left.\log _{10}\right)$ & $\begin{array}{c}\text { CFU/ml }\left(\log _{10}\right) / \\
\text { gelatin capsule }\end{array}$ \\
\hline $\begin{array}{l}\text { Facultative organisms } \\
\text { Escherichia coli }\end{array}$ & 6.32 & \\
Group D Streptococcus & 6.49 & 5.20 \\
Alpha hemolytic Streptococcus & 6.29 & 5.37 \\
Obligate anaerobes & & 5.17 \\
Clostridium perfringes & 8.05 & \\
Bacteroides thetaiotaomicron & 6.90 & 6.93 \\
Clostridium sordellii & 6.70 & 5.78 \\
Bacteroides vulgatus & 6.70 & 5.58 \\
Bacteroides caccae & 6.30 & 5.58 \\
Bacteroides fragilis & 6.00 & 5.18 \\
\end{tabular}

Cecal contents were harvested from meat-fed rats and contain microbial flora that approximate that of normal human colonic contents.

challenge with the mixed cecal contents inoculum a sixth treatment protocol (regimen 6) was employed in which animals were treated 24 $\mathrm{h}$ before surgery as well as $4,24,48$, and $96 \mathrm{~h}$ after challenge.

Cell transfer experiments. Cell transfer experiments were performed as described $(15,16)$. Spleens were removed from PS A-treated or naive rats, counted using a Coulter FN counter (Coulter Electronics Inc., Hialeah, FL), and examined for viability by trypan blue exclusion. The preparation was enriched for $\mathrm{T}$ cells by passage over nylon wool columns ( $>95 \%$ pure T cells as assessed by FACS analysis). Purified $\mathrm{T}$ cells were then counted and adjusted to appropriate cell number ( 1 $\times 10^{7}$ animal ) prior to intra-cardiac transfer to animals $(0.2 \mathrm{ml})$. Animals were challenged with bacterial inocula $24 \mathrm{~h}$ later.

Statistical analyses. Fisher's Exact Test was used to calculate differences in abscess-inducing potential between experimental and control groups.

\section{Results}

PS A-mediated protection against abscess-inducing heterologous bacterial species. Animals received subcutaneous injections of PS A according to an extended schedule (regimen 1, Table II) that had been employed with earlier studies of the $B$. fragilis $\mathrm{CPC}(6,13,15)$. After administration of $10 \mu \mathrm{g}$ doses of PS A according to regimen 1, animals were challenged via the intraperitoneal route with various inocula containing bacteria commonly associated with abscesses found in humans (Table III). These inocula have been shown previously to induce abscesses in the animal model (4). Groups of animals were challenged with a monomicrobial culture of $B$. fragilis, or mixed inocula consisting of a combination of $B$. distasonis and $E$. faecalis, B. thetaiotaomicron and $E$. faecalis or $F$. varium and $E$. faecalis. In each case, prophylactic treatment with PS A conferred protection against abscess formation (Table III).

Effect of regimen on protective activity. Several regimens were then tested that varied the duration of PS A administration before challenge with $B$. fragilis (Table II). In these experiments, protocols included regimens of 3 wk (regimen 1), 2 weeks (regimen 2), $1 \mathrm{wk}$ (regimen 3 ) and a group of animals that were treated $24 \mathrm{~h}$ before challenge as well as 4 and $24 \mathrm{~h}$ post-challenge (regimen 4). With each regimen, groups of animals were given $10 \mu \mathrm{g} /$ injection of PS A, challenged with $B$. fragilis and $6 \mathrm{~d}$ later examined for abscess formation. Irrespec- 


\begin{tabular}{|c|c|c|c|c|c|c|c|c|c|c|c|c|c|}
\hline & \multicolumn{13}{|c|}{ Days } \\
\hline & -28 & & -21 & & -14 & & -7 & -1 & 0 & +1 & +2 & +4 & +6 \\
\hline Regimen 1 & & +++ & & +++ & & +++ & & + & & & & & \\
\hline Regimen 2 & & & & +++ & & +++ & & + & & & & & \\
\hline Regimen 3 & & & & & & +++ & & + & & & & & \\
\hline Regimen 4 & & & & & & & & + & $+^{*}$ & + & & & \\
\hline Regimen 5 & & & & & & & & & $+*$ & + & + & & \\
\hline Regimen 6 & & & & & & & & + & $+^{*}$ & + & + & + & \\
\hline
\end{tabular}

* $1 \mathrm{~h}$ after challenge. + indicates administration of PS A at day pre- or post-challenge. Challenge occurred at day zero. Animals were killed at day 6 .

tive of the length of time animals were treated, fewer animals in each group possessed abscesses when compared to the salinetreated control group $(P<0.05)$. In addition, the protective activity was comparable in each of these groups. For regimens 1 through 4 the abscess rates were $26,18,32$, and $12 \%$, respectively, compared with the saline control group, which had an $80 \%$ abscess rate.

Determination of dose response for PS A administered by regimen 4. A dose response experiment was performed using the briefest of the four treatment regimens (regimen 4). In this experiment, significantly fewer animals receiving 10 or $1 \mu \mathrm{g} /$ injection had abscesses compared with saline-treated control animals (Table IV). However, animals receiving $0.1 \mu \mathrm{g} /$ injection of PS A were not significantly protected against abscess formation. $50 \%$ of animals receiving the lowest dose of PS A developed abscesses, while saline-treated animals had an abscess rate of $80 \%$.

Post-contamination treatment with PS A. To assess the ability of PS A to inhibit abscess induction following intraperitoneal contamination, animals were first challenged with $B$. fragilis and then treated with varying doses of PS A 1, 24, and $48 \mathrm{~h}$

Table III. PS A-mediated Protection Against Abscess Formation

\begin{tabular}{|c|c|c|c|}
\hline $\begin{array}{l}\text { Treatment* } \\
\quad(10 \mu \mathrm{g})\end{array}$ & Challenge inoculum & $\begin{array}{c}\text { Abscess } \\
\text { formation (No. } \\
\text { rats with } \\
\text { abscesses/ No. } \\
\text { tested) }\end{array}$ & $P$ value $^{+}$ \\
\hline Saline & B. fragilis & $15 / 18(83 \%)$ & - \\
\hline Saline & B. distasonis + E. faecalis & $16 / 18(89 \%)$ & - \\
\hline Saline & $\begin{array}{l}\text { B. thetaiotaomicron }+E \text {. } \\
\text { faecalis }\end{array}$ & $15 / 19(79 \%)$ & - \\
\hline Saline & $F$. varium + E. faecalis & $13 / 15(87 \%)$ & - \\
\hline PS A & B. fragilis & $1 / 19(5 \%)$ & $<0.0001$ \\
\hline PS A & B. distasonis + E. faecalis & $4 / 18(22 \%)$ & $<0.001$ \\
\hline PS A & $\begin{array}{l}\text { B. thetaiotaomicron }+E \text {. } \\
\text { faecalis }\end{array}$ & $2 / 19(11 \%)$ & $<0.0001$ \\
\hline PS A & $F$. varium $+E$. faecalis & $5 / 17(29 \%)$ & $<0.002$ \\
\hline \multicolumn{4}{|l|}{ S. pneumoniae } \\
\hline Type 3 CP & B. fragilis & $9 / 10(90 \%)$ & NS \\
\hline
\end{tabular}

* PS A given according to regimen 1 (three times a week for three weeks with one booster dose $24 \mathrm{~h}$ before challenge. ${ }^{\dagger}$ Compared with saline-treated controls receiving the same challenge inoculum. after challenge. Results from this experiment are also shown in Table IV. The 10 and $25 \mu \mathrm{g}$ dose failed to protect a significant number of animals compared with the saline-treated control group. However, treatment of infected animals with $50 \mu \mathrm{g} /$ injection of PS A yielded a significant level of protection against abscess formation compared with saline-treated controls $(P$ $=0.001$ ).

$T$ cell-mediated protection against abscess formation. Previously, it has been demonstrated that $T$ cells mediate protection against abscess formation induced by either viable $B$. fragilis or different abscess-inducing polysaccharides (14). Based on these findings, we investigated whether cell-mediated immune mechanisms also controlled the broadly protective activity exhibited by PS A in abrogating abscess formation by heterologous organisms commonly associated with intra-abdominal sepsis. Animals were treated with PS A $(10 \mu \mathrm{g} /$ injection $)$ according to regimen 3 and $\mathrm{T}$ cells isolated $24 \mathrm{~h}$ later. T cells $\left(1 \times 10^{7}\right)$ from PS A-treated or saline-treated animals were administered to naive recipients 24 hours before challenge with $B$. fragilis or with a mixed inoculum of $F$. varium and $E$. faecalis. Results from these experiments are shown in Table $\mathrm{V}$. Animals receiving $\mathrm{T}$ cells from saline-treated rats and challenged with $B$. fragilis or the $F$. varium and $E$. faecalis combination developed abscesses ( 85 and $87 \%$, respectively). However, transfer of $\mathrm{T}$ cells from PS A-treated animals to naive recipients yielded significant protection against abscess formation follow-

Table IV. PS A Dose Response and Post-Challenge Protection Against Abscess Formation

\begin{tabular}{|c|c|c|c|c|}
\hline $\begin{array}{l}\text { Treatment } \\
\text { regimen* }\end{array}$ & $\begin{array}{l}\text { Dose } \\
(\mu \mathrm{g})\end{array}$ & $\begin{array}{l}\text { Challenge } \\
\text { inoculum }\end{array}$ & $\begin{array}{c}\text { Abscess } \\
\text { formation (No. } \\
\text { rats with } \\
\text { abscesses/ No. } \\
\text { tested) }\end{array}$ & $\begin{array}{c}P \\
\text { value }^{\ddagger}\end{array}$ \\
\hline Saline & - & B. fragilis & $8 / 10(80 \%)$ & - \\
\hline Regimen 4 & 10 & B. fragilis & $5 / 19(26 \%)$ & 0.02 \\
\hline Regimen 4 & 1 & B. fragilis & $6 / 19(25 \%)$ & 0.02 \\
\hline Regimen 4 & 0.1 & B. fragilis & $10 / 20(50 \%)$ & NS \\
\hline Regimen 5 & 50 & B. fragilis & $3 / 19(16 \%)$ & 0.001 \\
\hline Regimen 5 & 25 & B. fragilis & $9 / 17(53 \%)$ & NS \\
\hline Regimen 5 & 10 & B. fragilis & $6 / 10(60 \%)$ & NS \\
\hline
\end{tabular}

* Administration of PS A as outlined in Table II. ${ }^{\ddagger}$ Compared with saline-treated controls. 
Table V. T cell-mediated Protection Against Abscess Formation

\begin{tabular}{|c|c|c|c|}
\hline $\begin{array}{l}\text { Treatment } \\
(10 \mu \mathrm{g})\end{array}$ & Challenge inoculum* & $\begin{array}{c}\text { Abscess } \\
\text { formation (No. } \\
\text { rats with } \\
\text { abscesses/No. } \\
\text { tested) }\end{array}$ & $P$ value $^{\dagger}$ \\
\hline Saline & B. fragilis & $11 / 13(85 \%)$ & - \\
\hline Saline & F. varium $+E$. faecalis & $13 / 15(87 \%)$ & - \\
\hline PS $A^{8}$ & B. fragilis & $1 / 8(13 \%)$ & $<0.005$ \\
\hline PS A & $F$. varium $+E$. faecalis & $3 / 14(21 \%)$ & $<0.005$ \\
\hline
\end{tabular}

* Challenge inoculum given $24 \mathrm{~h}$ after $\mathrm{T}$ cell transfer. ${ }^{\ddagger}$ Compared with animals given $\mathrm{T}$ cells from saline-immunized rats and then challenged with inoculum. ${ }^{8} \mathrm{~T}$ cells harvested $24 \mathrm{~h}$ after last dose of PS A administered by Regimen 3 (Table II).

ing challenge with $B$. fragilis ( $13 \%$ abscess rate) or $F$. varium and $E$. faecalis ( $21 \%$ abscess rate).

Protection against challenge with cecal contents. To simulate fecal contamination of the human peritoneal cavity, an inoculum of cecal contents was surgically implanted into the peritoneal cavities of rats. Although isolated from the ceca of rats, this inoculum contained a microbial flora similar to that found in the human colon (Table I). This inocula yielded $\sim 50 \%$ mortality with all the survivors developing abscesses. Initially, the efficacy of PS A (50 $\mu \mathrm{g} /$ injection according to regimen 4) against abscess formation by the cecal inoculum was tested. However, only a small reduction in the rate of abscess formation in treated animals was observed (data not shown). Therefore, another treatment regimen (regimen 6) was devised to increase the number of treatments with PS A after implantation of the cecal contents inoculum. In these experiments, animals were treated with $50 \mu \mathrm{g}$ of PS A $24 \mathrm{~h}$ before surgery as well as 4 , 24,48 , and $96 \mathrm{~h}$ after surgery. After treatment according to regimen 6 , significantly fewer animals developed abscesses compared with animals treated with saline (Table VI, $48 \%$ vs. $100 \%, P<0.0001)$.

\section{Discussion}

The role of $B$. fragilis and other members of this genus in intraabdominal abscess formation has been well documented (1). Although reports vary widely as to their prevalence, these organisms are isolated from more than half of all intra-abdominal infections. Other anaerobes such as Fusobacteria spp., Clos-

Table VI. PS A-mediated Protection Against Abscesses Induced by Cecal Contents Inoclum

\begin{tabular}{|c|c|c|c|}
\hline $\begin{array}{c}\text { Treatment* } \\
(50 \mu \mathrm{g} / \text { injection })\end{array}$ & $\begin{array}{l}\text { Challenge } \\
\text { inoculum }\end{array}$ & $\begin{array}{c}\text { Abscess } \\
\text { formation (No. } \\
\text { rats with } \\
\text { abscesses/No. } \\
\text { tested) }\end{array}$ & $P$ value $^{\ddagger}$ \\
\hline Saline & Cecal contents & $18 / 18(100 \%)$ & - \\
\hline PS A (Regimen 6) & Cecal contents & $12 / 25(48 \%)$ & $<0.0001$ \\
\hline
\end{tabular}

* Animals were treated according to regimen 6 (Table II). ${ }^{\ddagger}$ Compared with saline-treated controls. tridia spp., and Peptostreptococcus spp. are also isolated from such cases, but with less frequency. Our earlier reports suggested that PS A possessed protective activity against intraabdominal abscess formation caused by $B$. fragilis. We have now investigated whether this polysaccharide could confer protection against antigenically distinct organisms that are commonly associated with abscess formation in the clinical setting.

The ability of PS A to protect rats against challenge was first tested with four different bacterial inocula previously shown to induce intra-abdominal abscess formation in the animal model (4). One challenge inoculum consisted of viable $B$. fragilis alone, whereas the remaining inocula consisted of two different Bacteroides species and a $F$. varium strain each mixed with the facultative intestinal anaerobe, E. faecalis. Animals given PS A were protected against challenge with each of these different bacterial inocula. Protection against heterologous bacterial challenge suggested that suppression of a nonspecific immunologic mechanism regulating abscess induction could be responsible for the observed protective activity.

The aforementioned results were obtained using a treatment protocol of subcutaneous administration of PS A three times a week for three weeks with one booster dose $24 \mathrm{~h}$ before challenge. To address whether a brief immunomodulatory event rather than a sustained immunologic response was responsible for protection, four different treatment regimens of varying length were tested. Each of the four regimens used were effective in preventing abscesses including regimen 4 in which PS A was administered $24 \mathrm{~h}$ pre- as well as 4 and $24 \mathrm{~h}$ postchallenge. Dose response testing of PS A administered according to regimen 4 revealed that doses of 10 or $1 \mu \mathrm{g} /$ injection possessed equivalent protective activity. However, lowering the dose to $0.1 \mu \mathrm{g} /$ injection failed to significantly protect against abscess induction. The protective dose of PS A ( $1 \mu \mathrm{g})$ in these animal experiments was equivalent to $0.005 \mathrm{mg} / \mathrm{kg}$.

The development of abscesses associated with intra-abdominal sepsis usually occurs over several days after bacterial contamination of the peritoneal cavity. The kinetics of this host response is such that it provides a window of opportunity for post-contamination intervention to prevent abscess formation. Therefore, a therapeutic treatment regimen was used in which PS A was administered subsequent to contamination of the peritoneal cavity. While the standard dose of $10 \mu \mathrm{g} /$ injection failed to yield a significant level of protection, a higher dose of $50 \mu \mathrm{g} /$ injection was protective.

The ability of PS A to protect against abscesses induced by challenge with an inoculum consisting of cecal contents obtained from meat-fed rats was investigated. This challenge inoculum has been used previously in animals to simulate intraabdominal sepsis in humans after spillage of colonic contents into the peritoneal cavity $(4,5,6,17)$. The inoculum contains a wide variety and significant number of obligate and facultative organisms, and has been shown to induce a biphasic disease process that includes high rates of mortality followed by abscess formation in survivors. Therefore, this inoculum was diluted to yield a $50 \%$ mortality rate with $100 \%$ abscess formation in the survivors. The first attempt to protect animals against this large bacterial bolus using Regimen 4 at $50 \mu \mathrm{g}$ /injection was not successful, but suggested that a more extensive dose schedule might be used. Increasing the number of doses (from two to four) and duration (from two to four days) of treatment with PS A after peritoneal contamination resulted in significant protection against abscess formation. 
The role of $\mathrm{T}$ cells in induction of and protection against abscess formation by $B$. fragilis has been documented $(6,14$, 16). The present study demonstrated that $T$ cells were responsible not only for protection against $B$. fragilis, but for the nonspecific protective activity exhibited against different bacterial species capable of abscess formation in the animal model. The evidence presented indicates that PS A has immunomodulatory activity that is mediated by $\mathrm{T}$ cells or $\mathrm{T}$ cell-derived factors. However, this activity is unusual in that PS A does not elicit an antigen-specific anamnestic cell-mediated immune response. Rather, the protective activity is effective against structurally dissimilar antigens (either polysaccharides or intact bacteria) that share the ability to promote abscess formation. Previous studies have shown that $B$. fragilis $\mathrm{CPC}$ failed to consistently protect animals against abscess formation by different bacterial species $(6,7,13)$. It is clear that purification of PS A component from the CPC allows for the use of a more potent polysaccharide that is capable of protecting against $B$. fragilis as well as other bacterial species that cause abscess formation.

$\mathrm{T}$ cells with a suppressor phenotype comprise part of a cellular circuit that prevents abscess induction by $\boldsymbol{B}$. fragilis and lysates of these cells also transfer this protective activity $(7,16)$. We hypothesize that perhaps direct interaction of PS A with $T$ cells regulates cell activation or suppression that in turn results in the interruption of the immunologic events leading to abscess formation. Studies are currently underway to assess putative PS A-T cell interactions as well as the role of $\mathrm{T}$ cell-derived soluble mediators involved in this process.

B. fragilis PS A may be clinically useful in preventing abscess formation in the setting of intra-abdominal infections. Prophylaxis before abdominal or pelvic surgery with a high risk of peritoneal contamination is one situation where PS A administration may be considered for clinical testing. PS A has activity against a range of organisms that are most commonly isolated from these cases and exhibits protective activity against abscess formation caused by the cecal contents inoculum obtained from rats. The finding that protection can be afforded by administration of PS A following challenge with $B$. fragilis is clinically relevant when colonic spillage occurs in the setting of trauma or mucosal disruption. In the case of abscess formation subsequent to fecal contamination, the slow development of this tissue response provides a window of opportunity for PS A to perform its suppressive effect on abscess formation. Currently, we are evaluating the use of PS A for therapeutic protection against abscess formation subsequent to challenge with the cecal contents inoculum. Further testing of this polysaccharide is required to assess its potential for use in preventing abscess formation associated with clinical cases of intra-abdominal sepsis.

\section{Acknowledgments}

The authors would like to thank James Christian for expert technical assistance.

\section{References}

1. Nichols, R. L. 1985. Intraabdominal Infections: an Overview. Rev. Infect. Dis. 7:S709-S715.

2. Gorbach, S. L., and J. G. Bartlett. 1974. Anaerobic infections. N. Engl. J. Med. 290:1177-1184.

3. Polk, B. F., and D. L. Kasper. 1977. Bacteroides fragilis subspecies in clinical isolates. Ann. Int. Med. 86:569-571.

4. Onderdonk, A. B., J. G. Bartlett, T. Louie, N. Sullivan-Seigler, and S. L. Gorbach. 1976. Microbial synergy in experimental intra-abdominal abscess. Infect. Immun. 13:22-26.

5. Onderdonk, A. B., D. L. Kasper, R. L. Cisneros, and J. G. Bartlett. 1977. The capsular polysaccharide of Bacteroides fragilis as a virulence factor: comparison of the pathogenic potential of encapsulated and unencapsulated strains. $J$. Infect. Dis. 136:82-89.

6. Onderdonk, A. B., R. B. Markham, D. F. Zaleznik, R. L. Cisneros, and D. L. Kasper. 1982. Evidence for T cell-dependent Immunity to Bacteroides fragilis in an intraabdominal abscess model. J. Clin. Invest. 69:9-16.

7. Zaleznik, D. F., R. W. Finberg, M. E. Shapiro, A. B. Onderdonk, and D. L. Kasper. 1985. A soluble suppressor $T$ cell factor protects against experimental intraabdominal abscesses. J. Clin. Invest. 75:1023-1027.

8. Pantosti, A., A. O. Tzianabos, A. B. Onderdonk, and D. L. Kasper. 1991. Immunochemical characterization of two surface polysaccharides of Bacteroides fragilis. Infect. Immun. 59:2075-2082.

9. Baumann, H., A. O. Tzianabos, J.-R. Brisson, D. L. Kasper and H. J. Jennings. 1992. Structural elucidation of two capsular polysaccharides from one strain of Bacteroides fragilis using resolution NMR spectroscopy. Biochemistry. 31:4081-4089.

0. Tzianabos, A. O., A. Pantosti, H. Baumann, J. R. Brisson, H. J. Jennings, and D. L. Kasper. 1992. The capsular polysaccharide of Bacteroides fragilis comprises two ionically linked polysaccharides. J. Biol. Chem. 267:18230-18235. 11. Pantosti, A., A. O. Tzianabos, B. G. Reinap, A. B. Onderdonk and D. L. Kasper. 1993. Bacteroides fragilis strains express multiple capsular polysaccharides. J. Clin. Microbiol. 31:1850-1855.

12. Tzianabos, A. O., A. B. Onderdonk, B. Rosner, R. L. Cisneros, and D. L. Kasper. 1993. Structural features of polysaccharides that induce intra-abdominal abscesses. Science (Wash. DC). 262:416-419.

13. Kasper, D. L., A. B. Onderdonk, J. Crabb, and J. G. Bartlett. 1979. Protective efficacy of immunization with capsular antigen against experimental infection with Bacteroides fragilis. J. Infect. Dis. 140:724-731.

4. Tzianabos, A. O., A. B. Onderdonk, D. F. Zaleznik, R. S. Smith, and D. L. Kasper. 1994. Structural characteristics of polysaccharides that induce protection against intraabdominal abscess formation. Infect. Immun. 62:4881-4886.

15. Shapiro, M. E., A. B. Onderdonk, D. L. Kasper, and R. W. Finberg. 1982. Cellular immunity to Bacteroides fragilis capsular polysaccharide. J. Exp. Med. 154:1188-1197.

16. Shapiro, M. E., D. L. Kasper, D. F. Zaleznik, S. Spriggs, A. B. Onderdonk, and R. W. Finberg. 1986. Cellular control of abscess formation: role of $T$ cells in the regulation of abscesses formed in response to Bacteroides fragilis. J. Immunol. 137:341-346.

17. Onderdonk, A. B., W. M. Weinstein, N. M. Sullivan, J. G. Bartlett, and S. L. Gorbach. 1974. Experimental intra-abdominal abscesses in rats: quantitative bacteriology of infected animals. Infect. Immun. 10:1256-1259. 